\title{
MicroRNA-181a Suppresses Progestin-Stimulated Breast Cancer Cell Growth
}

\author{
Xue Li ${ }^{1}$, Chun Yang ${ }^{2}$, Xiangyan Ruan' ${ }^{1}$ Stéphane Croteau ${ }^{2}$, Pierre Hardy ${ }^{2 *}$ \\ 1Department of Gynecological Endocrinology, Beijing Obstetrics and Gynecology Hospital, Capital Medical University, Beijing, China \\ 2Departments of Medicine, Pediatrics, Pharmacology and Physiology, University of Montreal, Montreal, Quebec, Canada
}

\section{Article Info}

\section{Article Notes}

Received: July 23, 2018

Accepted: September 4, 2018

\section{*Correspondence:}

Dr. Pierre Hardy, MD, PhD, Research Center of CHU SainteJustine, 3175 Côte-Sainte-Catherine, Room 2714 Montréal, Québec, H3T 1C5, Canada; Telephone No: (514) 345-4931 (ext. 3656);

E-mail: pierre.hardy@recherche-ste-justine.qc.ca.

(C) 2018 Hardy P. This article is distributed under the terms of the Creative Commons Attribution 4.0 International License.

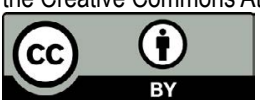

\section{Keywords:}

microrna-181a

Breast Cancer

Progestin

PGRMC1

Apoptosis

Norethisterone

\section{ABSTRACT}

Despite all our efforts, breast cancer remains a major public health problem threatening women's health all around the world. The morbidity of breast cancer is rising in most countries and is going to rise further over the next 20 years. Hormone treatment is widely used and it is the most efficient method of reducing menopausal symptoms or preventing abortion and pregnancy. However, multiple studies have demonstrated that hormones, especially synthetic progesterone, remain an indisputable risk factor for breast cancer. MicroRNAs are a group of endogenous small non-coding single strand RNAs which play regulatory roles in the initiation, development, and progression of different types of cancer. Evidence from multiple sources indicates that microRNA-181a exerts anti-breast cancer effects by inducing cancer cell death and preventing tumor invasion, infiltration and metastasis, etc. Our recent studies revealed that microRNA-181a not only suppresses breast cancer MCF7 cell growth but also abrogates progestin-provoked cell growth. In this review, several interesting aspects of hormone replacement therapy and the role of microRNA-181a during progestin treatment are discussed.

\section{Introduction}

In recent years, numerous studies have shown that microRNAs (miRNAs) play important roles in tumorigenesis, tumor progression, invasion and metastasis by silencing their target genes. We have also demonstrated that microRNA-181a (miR-181a) suppresses progestin-promoted breast cancer cell growth ${ }^{1}$. In this review, we focus on the interaction between miR-181a and progesterone receptor membrane component 1 (PGRMC1) in breast cancer cells after progestin stimulation. We also elucidated the possible underlying mechanisms of miR-181a which decreased the effect of norethisterone (NET) in breast cancer. The resulting data may provide a novel therapy for breast cancer treatment.

\section{Hormone Therapy Plays an Important Role in Postmenopausal Women}

According to the latest 2017 WHO statistics, life expectancy for Canadian women is 84.1 and the average age of menopause is 51 , which means that more than a third of women's lifetimes are spent in menopause. Menopause is a natural process but causes many pathological problems. For instance, it can increase the prevalence of coronary heart disease, osteoporosis, mood disorders, and sexual dysfunction ${ }^{2}$. Menopausal Hormone Therapy (MHT) is the most efficient therapy for menopausal symptoms and chronic diseases, but more than 50 studies have found that MHT can increase breast cancer risk ${ }^{3}$. 


\section{Progestin is Responsible for Increasing Breast Cancer Risk}

During MHT treatment, exogenous estrogens can stimulate endometrial cell proliferation, increasing the risk of endometrial cancer. There is a strong link between the use of estrogens and the occurrence of endometrial cancers. To reduce this risk, natural progesterone or synthetic progesterone (progestin) is used to stimulate atrophy or regression of endometrial lesions ${ }^{4-7}$. Nevertheless, on account of the short biological half-life of natural progesterone, its use is limited; therefore, progestins are widely used by women during MHT treatment ${ }^{8-10}$. However, the role of progestin in increasing breast cancer risk was reported by multiple clinical studies such as the Million Women Study (MWS) and Women's Health Initiative (WHI). They have demonstrated that combined estrogenprogestin therapy is associated with an increased risk of developing breast cancer ${ }^{11,12}$. Various progestins can exert different effects on breast cancer cell proliferation. Among the 9 selected types of natural progesterone and progestins, NET is the most potent progestin for promoting breast cancer cell proliferation ${ }^{13}$. In addition, we found that NET stimulated breast cancer cell viability of MCF-7 (ER+/PR+/ HER2-) and T-47D (ER+/PR+/HER2-) in a dose-dependent manner ${ }^{1}$.

\section{PGRMC1 Mediates Progestin-Stimulated Effect on Breast Cancer}

PGRMC1 is approximately a $25 \mathrm{kDa}$ protein. As a nonclassical membrane localized progesterone receptor, PGRMC1 binds progestogen with medium to high affinity ${ }^{14-16}$. Compelling evidence has accumulated showing that PGRMC1 contributes to cancer progression. Firstly, its expression was found up-regulated in tumors of the breast, thyroid, colon, ovary, cervix, and lung ${ }^{17-21}$. Secondly, PGRMC1 exhibited several cellular functions involved in the progression of several cancers. For example, PGRMC1 was found to be able to promote mitosis in spontaneously immortalized granulosa cells and human ovarian cancer cells $^{22}$, and to increase cell proliferation and migration of lung cancer cells as well ${ }^{23}$. Most interestingly, PGRMC1 mediated anti-apoptotic effect of progesterone in rat granulosa cells and in the immature rat ovary; this effect may be produced through interacting with plasminogen activator inhibitor RNA-binding protein- $1^{14,24-26}$. Depletion of PGRMC1 completely abrogated the anti-apoptosis effect of progesterone ${ }^{15,19}$. Furthermore, overexpression of PGRMC1 decreased the apoptosis rate in spontaneously immortalized granulosa cells ${ }^{26,27}$. In addition, a study indicated that PGRMC1 inhibitor abrogated the inhibiting effect of progesterone on proteins ratio of BAX/BCL-2 in neurons ${ }^{28}$, and overexpression of PGRMC1 elevated BCL-2 expression in ovarian cancer cells ${ }^{29}$. Another independent study indicated that the underlying mechanism of the anti- apoptotic effect of PGRMC1 is exerted through regulating expression of BH-3 only protein-Harakiri,aBCL-2interaction protein $^{30}$. Of note, BCL-2 functions as an oncogenic protein by inhibiting cell apoptosis. Overexpression of BCL-2 is common in breast cancer, especially in estrogen receptor (ER)-positive breast tumors ${ }^{31}$.

PGRMC1 is widely expressed in mammalian tissues and immortal mammalian cell lines. During the last 10 years, we have made contributions to elucidating the role of PGRMC1 in promoting breast cancer through hormone therapy. In vitro experiments showed that progestins can stimulate a proliferative response in PGRMC1overexpressing MCF-7 cells ${ }^{13}$, and in vivo NET-promoting tumor growth was observed in the xenografts bearing tumors developed from PGRMC1-overexpressed breast cancer cell T-47D ${ }^{32}$. Moreover, our clinical sample analysis revealed an increased expression of PGRMC1 in breast cancer tissues compared with surrounding normal tissues. The increased levels of PGRMC1 were positively correlated with aggressive phenotypes and poor prognosis ${ }^{33,34}$. All these studies suggest that PGRMC1 plays an important role in the progression of breast cancer, especially during hormone therapy. Along with these findings, our recent publication elucidated that NET markedly increases PGRMC1 expression in human breast cancer MCF-7 cells ${ }^{1}$.

\section{Anti-tumor effect of miRNA}

MiRNAs are a group of endogenous small non-coding regulatory RNAs, 21 to 24-nt in length. They silence their targeted genes at the post-transcriptional level. Usually, miRNAs interact with the targeted mRNAs within the $3^{\prime}$ untranslated regions (UTR) of target genes ${ }^{35}$. Aberrant expression of different miRNAs has been demonstrated in all kinds of cancer phenotypes ${ }^{36-38}$.

Our lab has been working on the pathophysiological roles of lymphocyte-derived microparticles (LMPs) for over ten years. LMPs were produced from human CEM T cells with treatment of actinomycin $\mathrm{D}^{39,40}$. We have strong evidence that LMPs play an important role in suppressing pathological angiogenesis and tumor progression ${ }^{41-46}$. In order to dissect the active components of LMPs, we performed miRNA sequence analysis and revealed that miR181a is one of the most plentiful miRNAs in LMPs. Several recent studies have suggested that miR-181a expression was downregulated in some types of human tumors such as lung cancer, oral squamous cell carcinoma, glioma etc. ${ }^{47-}$ ${ }^{52}$. Nevertheless, other studies indicated that miR-181a may perform oncomir activities by facilitating the metastasis and invasion of cancers ${ }^{53-55}$. Although contradictory data were reported, multiple clinical studies suggested that miR-181a expression was negatively correlated with breast cancer in late stages, with worse prognosis and with aggressive phenotypes ${ }^{54,56-58}$. The hypothesis that miR-181a may function as an anti-oncogene is further supported by 
our recent in vitro studies. We transfected the miR-181a-5p mimic into breast cancer cells and noticed that transitory overexpression of miR-181a significantly reduced cell viability of MCF-7 and T-47D breast cancer cell lines in a dose-dependent manner ${ }^{1}$.

\section{MiR-181a Inhibits Progestin-Stimulated Breast Cancer}

The mechanisms underlying the anti-tumor growth effect of miR-181a during NET treatment were investigated by our research group. We found that the apoptosis rate of the MCF-7 breast cancer cells in the miR-181a overexpression group was dramatically increased. Consistent with these results, we found a significantly decreased expression of the anti-apoptotic gene BCL-2 and a dramatic increase of the pro-apoptotic gene BAX and Caspase 9 in MCF-7 cells transfected with miR-181a. These results indicated a proapoptotic role of miR-181a involved in the anti-tumor growth effect. In addition to decreasing cell viability of MCF7, miR-181a also strongly abrogated the NET-induced cell viability of MCF-7 cells by increasing apoptosis ${ }^{1}$.

Apoptosis is a form of regulated cell death that is governed by pro- and anti-apoptotic members of the BCL2 protein family ${ }^{59}$. Lack of apoptosis can trigger oncogenic transformation at different stages and lead to increased tumor growth and survival during the metastatic process and chemotherapy. Increased expression of the antiapoptotic gene BCL-2 is found in many types of cancer. However, upregulation of BCL-2 expression can occur through all kinds of mechanisms. Therefore, preventing anti-apoptotic gene BCL-2 expression seems primordial in treating certain types of cancer. Activation of BCL-2associated $\mathrm{X}$ protein (BAX) results in mitochondrial outer membrane permeabilization, which is a necessary step for releasing small hemeprotein to activate the Caspase 3 and Caspase $7^{60}$. Because the MCF-7 cell lacks functional Caspase $3^{61}$, mitochondrial-dependent apoptosis proceeds via Caspase 7 and Caspase 9 activation. This is in keeping with an increase of Caspase 9 in miR-181a treated human breast cancer cells ${ }^{1}$. We have shown that miR-181a downregulated the expression of apoptosis related gene expression. Among these genes, BCL-2 is a direct target of miR-181a, which has been verified by luciferase assay in breast cancer cells ${ }^{62}$. In addition, PGRMC1 expression was decreased in the miR-181a overexpressing breast cancer cells. Furthermore, miR-181a attenuated NET-stimulated PGRMC1 expression in progesterone receptor positive and negative breast cancer cells ${ }^{1}$, which indicates that miR-181a probably exerts a pro-apoptotic effect through downregulation of PGRMC1 in these cells. These data lead us to assume the existence of functional interactions between PGRMC1 and miR-181a, although it has not been shown that they have a direct physical interaction ${ }^{63}$. As we mentioned above, since BCL-2 is a possible downstream effector of the anti-apoptotic action of PGRMC1 ${ }^{28-30}$, it is reasonable to speculate that the mechanism of miR181a increasing apoptosis of breast cancer cells is due to decreased expressions of BCL-2 and PGRMC1.

\section{Application Prospect of miR-181a as an Anti-Tumor Therapy}

In brief, miR-181a exerts strong anti-breast cancer activity, and this activity occurs through regulating the expression of apoptosis-related genes and PGRMC1. There is growing evidence that PGRMC1 contributes to cancer pathology. Up-regulated expression of PGRMC1 was found in a variety of tumors ${ }^{17-21}$. PGRMC1 facilitates the degradation of doxorubicin through its ability to bind CyP45020, which paves the way for speculation that a similar effect may be involved in the PGRMC1 mediated resistance to chemotherapeutics. In addition, overexpression of PGRMC1 can also lead to increased cancer cell proliferation and metastasis ${ }^{20}$. All these data suggest that PGRMC1 is of great importance to cancer progression. For the first time, we demonstrated that miR-181a can reduce PGRMC1 expression. Thus, miR-181a may develop into a novel therapeutic strategy not only for breast cancer treatment but also for other types of cancer overexpressing PGRMC1.

We have reported that miR-181a attenuates MCF-7 and MDA-MB-231 cell viability by reducing the upregulated protein expression of PGRMC1 induced by NET ${ }^{1}$. However, there are many other kinds of progestins which are widely used during hormone therapy. For instance, medroxyprogesterone acetate, which can also lead to the development of breast tumors in vivo and increase lymph node metastasis ${ }^{64}$. Further research could focus on the role miR-181a plays in reducing breast cancer cell viability provoked by different kinds of progestins. The aim would be to explore whether miR-181a has an extensive inhibitory effect on hormone-induced breast cancer.

\section{Acknowledgments}

This mini review was supported by an operating grant to Pierre Hardy from the Canadian Institutes of Health Research (362383).

\section{References}

1. Gu M, Wang L, Yang C, et al. Micro-RNA-181a suppresses progestinpromoted breast cancer cell growth. Maturitas. 2018; 114: 60-66.

2. Faubion SS, Kuhle CL, Shuster LT, et al. Long-term health consequences of premature or early menopause and considerations for management. Climacteric. 2015; 18(4): 483-91.

3. Mueck AO, Ruan X. Benefits and risks during HRT: main safety issue breast cancer. Horm Mol Biol Clin Investig. 2011; 5(2): 105-16.

4. Smith DC, Prentice R, Thompson DJ, et al. Association of exogenous estrogen and endometrial carcinoma. N Engl J Med. 1975; 293(23): 1164-7.

5. Ziel HK, Finkle WD. Increased risk of endometrial carcinoma among users of conjugated estrogens. N Engl J Med. 1975; 293(23): 1167-70. 
6. Grady D, Gebretsadik T, Kerlikowske K, et al. Hormone replacement therapy and endometrial cancer risk: a meta-analysis. Obstet Gynecol. 1995; 85(2): 304-13.

7. Crosbie EJ, Zwahlen M, Kitchener HC, et al. Body mass index, hormone replacement therapy, and endometrial cancer risk: a meta-analysis. Cancer Epidemiol Biomarkers Prev. 2010; 19(12): 3119-30.

8. Louw-du TR, Perkins MS, Hapgood JP, et al. Comparing the androgenic and estrogenic properties of progestins used in contraception and hormone therapy. Biochem Biophys Res Commun. 2017; 491(1): 140146.

9. Schindler AE, Campagnoli C, Druckmann R, et al. Classification and pharmacology of progestins. Maturitas. 2003; 46 Suppl 1: S7-S16.

10. Africander D, Verhoog N, Hapgood JP. Molecular mechanisms of steroid receptor-mediated actions by synthetic progestins used in HRT and contraception. Steroids. 2011; 76(7): 636-52.

11. Beral V. Breast cancer and hormone-replacement therapy in the Million Women Study. Lancet. 2003; 362(9382): 419-27.

12. Chlebowski RT, Hendrix SL, Langer RD, et al. Influence of estrogen plus progestin on breast cancer and mammography in healthy postmenopausal women: the Women's Health Initiative Randomized Trial. JAMA. 2003; 289(24): 3243-53.

13. Ruan X, Neubauer H, Yang Y, et al. Progestogens and membraneinitiated effects on the proliferation of human breast cancer cells. Climacteric. 2012; 15(5): 467-72.

14. Peluso JJ, Pappalardo A, Losel R, et al. Progesterone membrane receptor component 1 expression in the immature rat ovary and its role in mediating progesterone's antiapoptotic action. Endocrinology. 2006; 147(6): 3133-40.

15. Peluso JJ, Romak J, Liu X. Progesterone receptor membrane component-1 (PGRMC1) is the mediator of progesterone's antiapoptotic action in spontaneously immortalized granulosa cells as revealed by PGRMC1 small interfering ribonucleic acid treatment and functional analysis of PGRMC1 mutations. Endocrinology. 2008; 149(2): 534-43.

16. Meyer C, Schmid R, Scriba PC, et al. Purification and partial sequencing of high-affinity progesterone-binding site(s) from porcine liver membranes. Eur J Biochem. 1996; 239(3): 726-31.

17. Hampton KK, Stewart R, Napier D, et al. PGRMC1 Elevation in Multiple Cancers and Essential Role in Stem Cell Survival. Adv Lung Cancer (Irvine). 2015; 4(3): 37-51.

18. Crudden G, Loesel R, Craven RJ. Overexpression of the cytochrome p450 activator hpr6 (heme-1 domain protein/human progesterone receptor) in tumors. Tumour Biol. 2005; 26(3): 142-6.

19. Peluso JJ, Liu X, Saunders MM, et al. Regulation of ovarian cancer cell viability and sensitivity to cisplatin by progesterone receptor membrane component-1. J Clin Endocrinol Metab. 2008; 93(5): 15929.

20. Kabe Y, Nakane T, Koike I, et al. Haem-dependent dimerization of PGRMC1/Sigma-2 receptor facilitates cancer proliferation and chemoresistance. Nat Commun. 2016; 7: 11030.

21. Lösel RM, Besong D, Peluso JJ, et al. Progesterone receptor membrane component 1--many tasks for a versatile protein. Steroids. 2008; 73(9-10): 929-34.

22. Lodde V, Peluso JJ. A novel role for progesterone and progesterone receptor membrane component 1 in regulating spindle microtubule stability during rat and human ovarian cell mitosis. Biol Reprod. 2011; 84(4): 715-22.

23. Ahmed IS, Rohe HJ, Twist KE, et al. Progesterone receptor membrane component 1 (Pgrmc1): a heme-1 domain protein that promotes tumorigenesis and is inhibited by a small molecule. J Pharmacol Exp Ther. 2010; 333(2): 564-73.
24. Peluso JJ, Pappalardo A, Losel R, et al. Expression and function of PAIRBP1 within gonadotropin-primed immature rat ovaries: PAIRBP1 regulation of granulosa and luteal cell viability. Biol Reprod. 2005; 73(2): 261-70.

25. Cahill MA. Progesterone receptor membrane component 1: an integrative review. J Steroid Biochem Mol Biol. 2007; 105(1-5): 16-36.

26. Peluso JJ, Liu X, Gawkowska A, et al. Progesterone inhibits apoptosis in part by PGRMC1-regulated gene expression. Mol Cell Endocrinol. 2010; 320(1-2): 153-61.

27. Mansouri MR, Schuster J, Badhai J, et al. Alterations in the expression, structure and function of progesterone receptor membrane component-1 (PGRMC1) in premature ovarian failure. Hum Mol Genet. 2008; 17(23): 3776-83.

28. Qin Y, Chen Z, Han X, et al., Progesterone attenuates Abeta(25-35)induced neuronal toxicity via JNK inactivation and progesterone receptor membrane component 1-dependent inhibition of mitochondrial apoptotic pathway. J Steroid Biochem Mol Biol. 2015; 154: 302-11.

29. Zhu X, Ji M, Han Y, et al. PGRMC1-dependent autophagy by hyperoside induces apoptosis and sensitizes ovarian cancer cells to cisplatin treatment. Int J Oncol. 2017; 50(3): 835-846.

30. Will EA, Liu X, Peluso JJ. Peluso, AG 205, a progesterone receptor membrane component 1 antagonist, ablates progesterone's ability to block oxidative stress-induced apoptosis of human granulosa/luteal cellsdagger. Biol Reprod. 2017; 96(4): 843-854.

31. Oakes SR, Vaillant F, Lim E, et al. Sensitization of BCL-2-expressing breast tumors to chemotherapy by the BH3 mimetic ABT-737. Proc Natl Acad Sci U S A. 2012; 109(8): 2766-71.

32. Zhao $\mathrm{Y}$, Ruan $\mathrm{X}$, Wang $\mathrm{H}$, et al. The presence of a membrane-bound progesterone receptor induces growth of breast cancer with norethisterone but not with progesterone: A xenograft model. Maturitas. 2017; 102: 26-33.

33. Ruan X, Zhang Y, Mueck AO, et al. Increased expression of progesterone receptor membrane component 1 is associated with aggressive phenotype and poor prognosis in ER-positive and negative breast cancer. Menopause. 2017; 24(2): 203-209.

34. Zhang Y, Ruan X, Willibald M, et al. May progesterone receptor membrane component 1 (PGRMC1) predict the risk of breast cancer? Gynecol Endocrinol. 2016; 32(1): 58-60.

35. Bartel DP. MicroRNAs: genomics, biogenesis, mechanism, and function. Cell. 2004; 116(2): 281-97.

36. Macfarlane LA, Murphy PR. MicroRNA: Biogenesis, Function and Role in Cancer. Curr Genomics. 2010; 11(7): 537-61.

37. Zhao S, Han J, Zheng L, et al., MicroRNA-203 Regulates Growth and Metastasis of Breast Cancer. Cell Physiol Biochem. 2015; 37(1): 35-42.

38. Chen J, Ge B, Wang Y, et al. Biochanin A promotes proliferation that involves a feedback loop of microRNA-375 and estrogen receptor alpha in breast cancer cells. Cell Physiol Biochem. 2015; 35(2): 63946.

39. Martin S, Tesse A, Hugel B, et al., Shed membrane particles from T lymphocytes impair endothelial function and regulate endothelial protein expression. Circulation. 2004; 109(13): 1653-9.

40. Tesse A, Martínez MC, Hugel B, et al. Upregulation of proinflammatory proteins through NF-kappaB pathway by shed membrane microparticles results in vascular hyporeactivity. Arterioscler Thromb Vasc Biol. 2005; 25(12): 2522-7.

41. Yang C, Mwaikambo BR, Zhu T, et al. Lymphocytic microparticles inhibit angiogenesis by stimulating oxidative stress and negatively regulating VEGF-induced pathways. Am J Physiol Regul Integr Comp Physiol. 2008; 294(2): R467-76. 
42. Yang C, Gagnon C, Hou X, et al., Low density lipoprotein receptor mediates anti-VEGF effect of lymphocyte T-derived microparticles in Lewis lung carcinoma cells. Cancer Biol Ther. 2010; 10(5): 448-56.

43. Tahiri H, Yang C, Duhamel F, et al., p75 neurotrophin receptor participates in the choroidal antiangiogenic and apoptotic effects of T-lymphocyte-derived microparticles. Invest Ophthalmol Vis Sci. 2013; 54(9): 6084-92.

44. Yang C, Xiong W, Qiu Q, et al. Role of receptor-mediated endocytosis in the antiangiogenic effects of human T lymphoblastic cell-derived microparticles. Am J Physiol Regul Integr Comp Physiol. 2012; 302(8): R941-9.

45. Yang C, Xiong W, Qiu Q et al. Anti-proliferative and anti-tumour effects of lymphocyte-derived microparticles are neither species- nor tumour-type specific. J Extracell Vesicles. 2014; 3.

46. Tahiri H, Omri S, Yang C, et al. Lymphocytic Microparticles Modulate Angiogenic Properties of Macrophages in Laser-induced Choroidal Neovascularization. Sci Rep. 2016; 6: 37391.

47. Chen G, Zhu W, Shi D, et al. MicroRNA-181a sensitizes human malignant glioma U87MG cells to radiation by targeting Bcl-2. Oncol Rep. 2010; 23(4): 997-1003.

48. Shi L, Cheng Z, Zhang J, et al. hsa-mir-181a and hsa-mir-181b function as tumor suppressors in human glioma cells. Brain Res. 2008; 1236: 185-93.

49. Shin KH, Bae SD, Hong HS, et al. miR-181a shows tumor suppressive effect against oral squamous cell carcinoma cells by downregulating K-ras. Biochem Biophys Res Commun. 2011; 404(4): 896-902.

50. Ciafrè SA, Galardi S, Mangiola A, et al. Extensive modulation of a set of microRNAs in primary glioblastoma. Biochem Biophys Res Commun. 2005; 334(4): 1351-8.

51. Gao W, Yu Y, Cao H, et al. Deregulated expression of miR-21, miR143 and miR-181a in non small cell lung cancer is related to clinicopathologic characteristics or patient prognosis. Biomed Pharmacother. 2010; 64(6): 399-408.

52. Cao Y, Zhao D, Li P, et al. MicroRNA-181a-5p Impedes IL-17-Induced Nonsmall Cell Lung Cancer Proliferation and Migration through Targeting VCAM-1. Cell Physiol Biochem. 2017; 42(1): 346-356.
53. Wang Y, Yu Y, Tsuyada A, et al. Transforming growth factor-beta regulates the sphere-initiating stem cell-like feature in breast cancer through miRNA-181 and ATM. Oncogene. 2011; 30(12): 1470-80.

54. Zhu Y, Wu J, Li S, et al. The function role of miR-181a in chemosensitivity to adriamycin by targeting Bcl-2 in low-invasive breast cancer cells. Cell Physiol Biochem. 2013; 32(5): 1225-37.

55. Yang CC, Hung PS, Wang PW, et al. miR-181 as a putative biomarker for lymph-node metastasis of oral squamous cell carcinoma. J Oral Pathol Med. 2011; 40(5): 397-404.

56. Berber U, Yilmaz I, Narli G, et al. miR-205 and miR-200c: Predictive Micro RNAs for Lymph Node Metastasis in Triple Negative Breast Cancer. J Breast Cancer. 2014; 17(2): 143-8.

57. Wang B, Li J, Sun M, et al. miRNA expression in breast cancer varies with lymph node metastasis and other clinicopathologic features. IUBMB Life. 2014; 66(5): 371-7.

58. Li Y, Kuscu C, Banach A, et al., miR-181a-5p Inhibits Cancer Cell Migration and Angiogenesis via Downregulation of Matrix Metalloproteinase-14. Cancer Res. 2015; 75(13): 2674-85.

59. Meier P, Finch A, Evan G. Apoptosis in development. Nature. 2000; 407(6805): 796-801.

60. Hotchkiss RS. Cell death. N Engl J Med. 2009; 361(16): 1570-83.

61. Galluzzi L, Kepp O, Trojel-Hansen C, et al. Mitochondrial control of cellular life, stress, and death. Circ Res. 2012. 111(9): 1198-207.

62. $\mathrm{Zhu} \mathrm{Y,Wu} \mathrm{J,} \mathrm{LiS,} \mathrm{et} \mathrm{al.} \mathrm{The} \mathrm{function} \mathrm{role} \mathrm{of} \mathrm{miR-181a} \mathrm{in} \mathrm{chemosensitivity}$ to adriamycin by targeting Bcl-2 in low-invasive breast cancer cells. Cell Physiol Biochem. 2013; 32(5): 1225-37.

63. Niu J, Xue A, Chi Y, et al. Induction of miRNA-181a by genotoxic treatments promotes chemotherapeutic resistance and metastasis in breast cancer. Oncogene. 2016; 35(10): 1302-1313.

64. Goyette S, Liang Y, Mafuvadze B, et al. Natural and synthetic progestins enrich cancer stem cell-like cells in hormone-responsive human breast cancer cell populations in vitro. Breast Cancer (Dove Med Press). 2017; 9: 347-357. 